ARTÍCULO DE INVESTIGACIÓN

\title{
FUNCIÓN DE LA IMPULSIVIDAD EN EL TRASTORNO POR CONSUMO DE SUSTANCIAS
}

\author{
IMPULSIVITY`S ROLE IN SUBSTANCE USE DISORDER
}

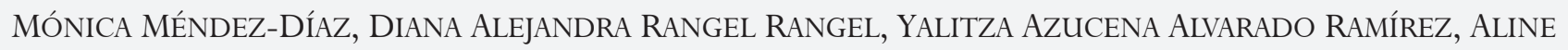
MENDOZA-MÉNDEZ, ${ }^{1}$ MÓNICA ANDREA HERRERA-SOlís, ${ }^{2}$ JaQUEline CORTÉS MORELOS, ${ }^{3}$ AlejANDRA EVELYN RUIZ-CONTRERAS Y OSCAR PROSPÉRO-GARCÍA.

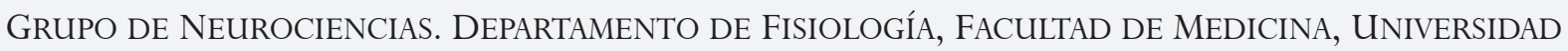
NACIONAL AUTÓNOMA DE MÉXiCO. ${ }^{1}$ LABORATORIO EFECTOS TERAPÉUTICOS DE LOS CANABINOIDES, SUbDiRECCIÓN DE INVESTIGACIÓN BIOMÉDICA, HosPitAl GENERAL DR. MANUEL GEA GONZÁLEZ.

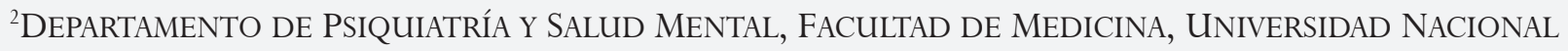

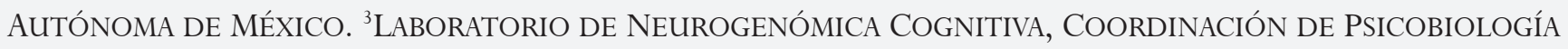
y NEUROCIENCIAS, FACULTAD DE PSICOlOGía. UNIVERSIDAD NACIONAL AUTÓNOMA DE MÉXICO.

\section{FECHA RECEPCIÓN: 21/05/2021 • FECHA ACEPTACIÓN: 22/06/2021}

\begin{abstract}
Para citar este artículo: Méndez-Díaz, M., Rangel, D., Alvarado, Y., Mendoza-Méndez, A., Herrera-Solís MA., Cortés, J. RuizContreras, A., \& Prospéro-García, O. (2021). Función de la impulsividad en el trastorno por consumo de sustancias. Psychologia, 15(1), 83-93. https://doi: 10.21500/19002386.5390.
\end{abstract}

\section{Resumen}

La proporción de usuarios de una sustancia de abuso que desarrolla problemas con su consumo (abuso o dependencia) representa solo una parte de esta población. En México, el 63.8 \% de la población consume alcohol, y de ellos, el $15 \%$ desarrolla algún trastorno por consumo de alcohol (TCA). Se ha observado una relación causal entre el trastorno por consumo de sustancias (TCS) y la falta de autocontrol. Es decir, satisfacer necesidades de manera

1 Mónica Méndez-Díaz (ORCID: 0000-0002-0949-3070) * Correspondencia: Dra. Mónica Méndez Díaz. Departamento de Fisiología, Facultad de Medicina, Universidad Nacional Autónoma de México. Apdo. Postal 70-250, México, D. F. 04510. México. Teléfono: (52-55) 623 2509 Fax: (52-55) 623 2241.mendezm@unam.mx

2 Diana Alejandra Rangel Rangel (ORCID: 0000-0002-1762-0017), Yalitza Azucena Alvarado Ramírez (ORCID: 0000-0002-5971-5153), Aline Mendoza Méndez (ORCID: 0000-0002-4265-8869)

3 Andrea Mónica Herrera Solís (ORCID: 0000-0001-7400-3325), Jaqueline Cortes Morelos (ORCID: 0000-0003-0320-4802), Alejandra Evelyn RuizContreras (ORCID: 0000-0002-9829-7636), Oscar Prospéro-García (ORCID: 0000-0003-4936-3139)

Agradecimientos. Este trabajo fue apoyado por la Dirección General de Asuntos del Personal Académico-Programa de Apoyo a Proyectos de Investigación e Innovación Tecnológica, Universidad Nacional Autónoma de México (DGAPA-PAPIIT-UNAM) Donativos: IN217918 a AERC, IN218620 a OPG e IA205218 a MMD. 
impulsiva, v. gr., consumir una droga sin evaluar las consecuencias. La corteza prefrontal (CPF) es el principal sustrato neuroanatómico del autocontrol y característicamente la CPF alcanza la madurez alrededor de los 30 años, sugieriendo que el autocontrol se alcanza despues de esta edad. Se ha propuesto que todos los grupos etarios que no han consolidado el uso del autocontrol son vulnerables al TCS. Similarmente ocurre con aquellos sujetos que por algún trastorno psiquiátrico tienen como característica una limitada función prefrontal. La CPF coordina una red subcortical cuya interacción depende de distintos sistemas de neurotransmisión, entre ellos, endocanabinoides. En este trabajo se revisó la función de la CPF y del sistema de endocanabinoides (sECB) y su relación con la vulnerabilidad a la adicción y otros trastornos psiquiátricos.

Palabras clave: Impulsividad, autocontrol, transtorno por uso de sustancias, enfermedades psiquiátricas.

\section{Abstract}

The proportion of users of a substance of abuse who develop problems with its use (abuse or dependence) represents only a part of this population. In Mexico, 63.8\% of the population consumes alcohol and only $15 \%$ of them develop an alcohol use disorder (AUD). A causal relation has been observed between substance use disorder (SUD) and the lack of self-control. Which means, satisfying needs in an impulsive way, v.gr. using a drug, without considering the consequences. The prefrontal cortex (PFC) is the main neuroanatomical substrate of self-control and characteristically reaches maturity around the age of 30, suggesting that self-control is reached after this age. We suggest that all age groups that have not consolidated the use of self-control are vulnerable to SUD. The same occurs with those who, due to a psychiatric disorder, have the characteristic of a limited prefrontal function. The PFC coordinates a subcortical network whose interaction depends on different neurotransmission systems among them, the endocannabinoids system (ECBs). In this work we will review the function of the PFC, the ECBs and its relationship with vulnerability to addiction and other psychiatric disorders.

Key words: Impulsivity, self-control, substance use disorder, psychiatric disorders

\section{Introducción}

El TCS es un trastorno psiquiátrico que provoca la búsqueda y el uso compulsivo de una o varias sustancias de abuso, a pesar de las consecuencias adversas que estas provoquen (Kalivas \& Volkow, 2005). Según el Manual de Diagnóstico Estadístico de los Trastornos Mentales V (DSM-V), para que un paciente sea diagnosticado con TCS debe manifestar las siguientes características: sentir un fuerte deseo por consumir la sustancia de abuso, tener dificultad para controlar su consumo, presentar síndrome de abstinencia cuando interrumpe o reduce el consumo de la misma, presentar tolerancia a su efectos, abandonar actividades que son ajenas al consumo, ocupar el mayor tiempo del día para conseguir y consumir la sustancia y persistir en su uso, a pesar de provocarle problemas laborales o sociales. En resumen, el TCS se asocia con una pérdida del control en el consumo de la(s) sustancia(s) (American Psychiatric Association, 2013). Algunos autores han propuesto que conductualmente el TCS es una serie de ciclos recurrentes de tres etapas: 1. atracones/intoxicaciones, 2. abstinencia/ afecto negativo y 3. preocupación/ansia, asociadas a cambios neurofisiológicos en los sistemas de recompensa, defensa y función ejecutiva (Koob \& Volkow, 2016).

En México, según la Encuesta Nacional de Consumo de Drogas Alcohol y Tabaco 2016-2017 (CONADIC, 2017), el 9.9 \% de la población de entre 12 y 65 años ha consumido alguna droga ilegal. Esta misma encuesta reporta que las drogas ilícitas más consumidas son la mariguana (8.6\%), cocaína (3.5\%) e inhalables (1.1\%). Por otro lado, el consumo nocivo y excesivo de alcohol se incrementó de 2011 a 2016 en un 93 \%. Igualmente, se reportó que, a partir de los diez años, 1 de cada 2 niños consumen alcohol, tomando hasta 20 copas por ocasión. Dentro de los adolescentes de quinto y sexto año de primaria existen más de 110000 sujetos con TCS (CONADIC, 2019; ENSANUT, 2018; Velázquez et al., 2016). En el 2016, 148 millones de personas, que representa el 2\% de la población mundial, presentó algún TCS (Institute for Health Metrics and Evaluation, 2018). 
En resumen, a pesar de que todos los humanos están expuestos a consumir drogas de abuso y poseen los mecanismos cerebrales que les permiten experimentar sus efectos placenteros, solo un porcentaje bajo de la población es vulnerable a padecer algún TCS. Por lo que resulta impresindible describir inicialmente los mecanismos cerebrales involucrados en el placer y, posteriormente, los involucrados en la decisión de consumo, para entender cómo su disfunción resulta en la expresión de algún TCS.

Figura 1. Sistema del placer

a

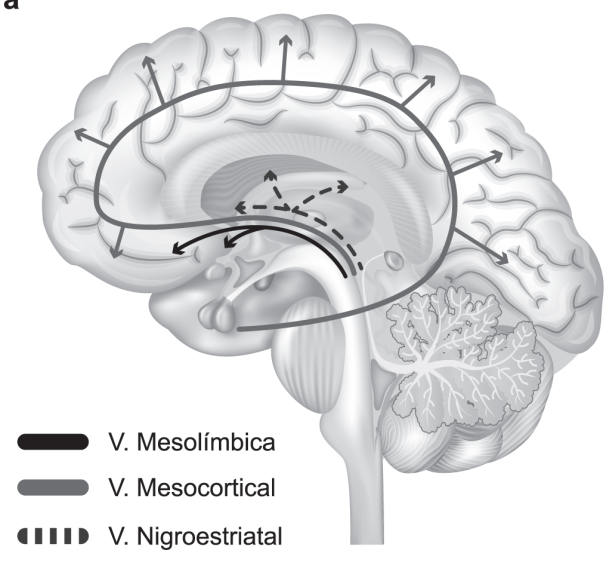

Nota: En la figura se muestran las principales vías dopaminérgicas, la vía mesolímbica es parte fundamental del sistema de motivación y recompensa. a) Vías dopaminérgicas. La vía mesocortical está involucrada en la regulación de los procesos cognitivos, aprendizaje y memoria, atención y emoción. La vía mesolímbica regula la motivación, placer, recompensa, búsqueda, emoción y adicción. La vía nigroestriatal está involucrada en el control del movimiento y estimulación sensorial. b) Liberación de DA tras la activación del ATV por diferentes reforzadores, a mayor liberación de DA, mayor sensación de placer. Esta figura se ha construido a partir de la información de Baik (2013); Kalivas y Volkow (2005); Koob y Volkow (2016). Fue creada por el Diseñador Sergio Broseta por instrucciones de los autores para los objetivos de esta publicación. b

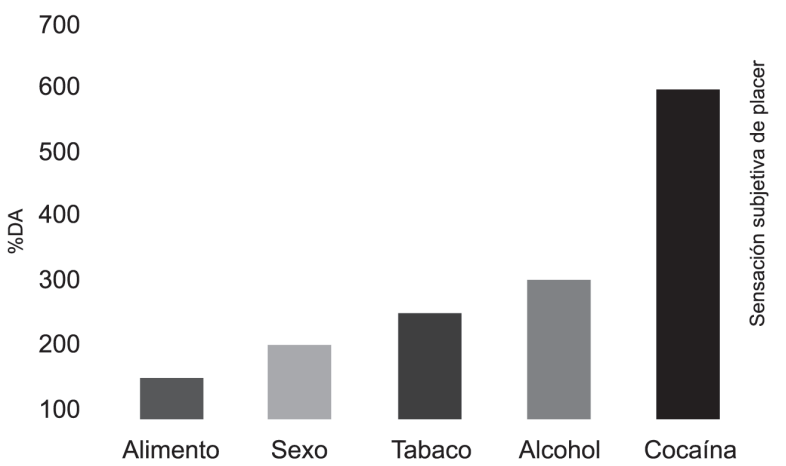

\section{Mecanismos cerebrales}

El del placer. La sensación placentera que experimenta el humano al comer, beber o tener sexo es regulada por diversas estructuras del sistema límbico y por neuronas dopaminérgicas, organizadas en un área llamada área tegmental ventral (ATV). Estas neuronas dopaminérgicas proyectan a diferentes núcleos cerebrales, constituyendo dos de las principales vías dopaminérgicas: la vía mesolímbica, que va del ATV al núcleo accumbens (NAc) y a la amígdala (AMI), y la vía mesocortical, que parte del ATV y proyecta a la CPF (Figura 1) (Méndez-Díaz et al., 2017).

$$
\text { Alimento sexo }
$$


funciones ejecutivas incluyen la inhibición de la respuesta conductual, v. gr., evitar conductas no adaptativas; la flexibilidad cognitiva, v. gr., aceptar que las otras personas piensan diferente, aceptar que lo que se piensa hoy puede cambiar mañana y aceptar que lo que se cree que esta ocurriendo puede ser diferente a lo que realmente está ocurriendo, y la memoria de trabajo, v. gr., actualizar nuestros conceptos conforme los eventos ocurren. Los procesos cognitivos son indispensables para llevar a cabo la mayor parte de las actividades diarias, principalmente las que involucran la creación de planes, la toma de decisiones, la solución de problemas y el autocontrol. La CPF recibe información de los sistemas sensoriales y motores, y los compara con eventos semejantes almacenados en la memoria de largo plazo (análisis) para generar la respuesta adaptativa en ese momento. Posee un sistema de neurotransmisión múltiple: dopaminérgico, serotoninérgico, colinérgico y endocanabinérgico (Amancio-Belmont et al., 2017; Tirapu-Ustárroz et al., 2008; Wędzony \& Chocyk, 2009).

A diferencia de otras estructuras, la CPF continúa desarrollándose después del nacimiento, principalmente durante la infancia y la adolescencia. Neurofuncionalmente, significa una disminución de la materia gris, mayor mielinización, comunicación sináptica eficiente y cambio en los sistemas de neurotransmisión (Dumontheil et al., 2008). Conductualmente implica el perfeccionamiento de las capacidades cognitivas mencionadas que nos permiten pasar de la respuesta impulsiva, que es la habilidad con la que nacemos, a la respuesta reflexiva para adaptar eficientemente el comportamiento a los cambios que pueden producirse en el entorno. Esta es la capacidad ejecutiva observada en la mayoría de los adultos a principios de la segunda década de vida (García-Molina et al., 2009) y la maduración cerebral alrededor de los 30 años. Es decir, fisiológicamente, niños y adolescentes carecen de autocontrol, lo que los deja a merced de comportamientos impulsivos que, a pesar de que son necesarios para conocer el mundo que los rodea, son riesgosos, por lo que los expone a consecuencias que atentan contra su integridad física y emocional, ya que típicamente rompen reglas, pelean, hurtan y consumen sustancias de abuso.

Con base en lo anterior, podemos señalar que los niños y adolescentes son vulnerables al desarrollo de un TCS, potencialmente por dos razones: la primera es que la exposición a sustancias de abuso a edades tempranas altera el subsecuente desarrollo del sistema nervioso central, y la segunda, es que la falta de maduración de la CPF interfiere con una toma de decisiones basada en las consecuencias. Es decir, aprender de lo que se hace. Básicamente, el riesgo de padecer un TCS disminuye conforme la CPF madura y se retrasa la exposición a drogas. Vale la pena insistir que no todas las CPFs alcanzan a "madurar", incluso después de los 30, perpetuando la falta de autocontrol y la practica de coductas riesgosas.

Como ya señalamos, el TCS se caracteriza particularmente por la pérdida del control en el consumo de las sustancias. A continuación, describiremos como la CPF y la red subcortical multiestructural regulan el autocontrol.

El del autocontrol. El autocontrol es un mecanismo adaptativo que nos permite regular la ejecución de conductas que satisfacen impulsos momentáneos, al evaluar las ganancias o consecuencias de llevar a cabo cierta conducta. Con el autocontrol el sujeto es capaz de analizar las ventajas de soslayar la obtención de un reforzador inmediato, que provee de un beneficio bajo o moderado con miras en la obtención de un reforzador en el largo plazo, pero que provee de un beneficio mayor. La función de este sistema es inhibir impulsos y tendencias de respuesta inmediata, que a la larga resulten no adaptativas. Como ya se dijo, est anatómicamente conformado por la CPF y la red subcorticalmulti-estructural. La comunicación entre estas estructuras ocurre de tres formas: la vía directa, la vía indirecta y la vía hiperdirecta (Aron \& Poldrack, 2006) (Figura 2). La vía directa facilita la ejecución de la conducta, mientras que las vías indirecta e hiperdirecta inhiben la conducta por diferentes mecanismos.

Adicionalmente, la habénula lateral ( $\mathrm{HbL}$ ) forma parte de esta red subcortical del control de impulsos, regulando la actividad del ATV. El globo pálido interno (GPi), llamado núcleo entopeduncular (EP) en la rata, que proyecta al tálamo en la vía indirecta, también proyecta a la $\mathrm{HbL}$, activándola por medio de glutamato (GLU). La HbL regula, por medio de una vía GLUérgica, a la región rostral medial tegmental o cola del AVT (tATV/RMTg), la cual es GABAérgica. La tATV regula a la porción anterior del ATV, inhibiéndola. Este sistema de regulación inhibidora de la conducta reduce o inhibe la 
búsqueda del reforzador y la sensación subjetiva de placer (Barrot et al., 2012). En el caso contrario, en el que dicho sistema se encuentra inhibido, se genera la conducta de búsqueda del reforzador placentero (Lammel et al., 2012). Sugiendo que la HbL es el puente de comunicación entre el gusto por el reforzador y la conducta de busqueda.

Figura 2.Sistema de autocontrol

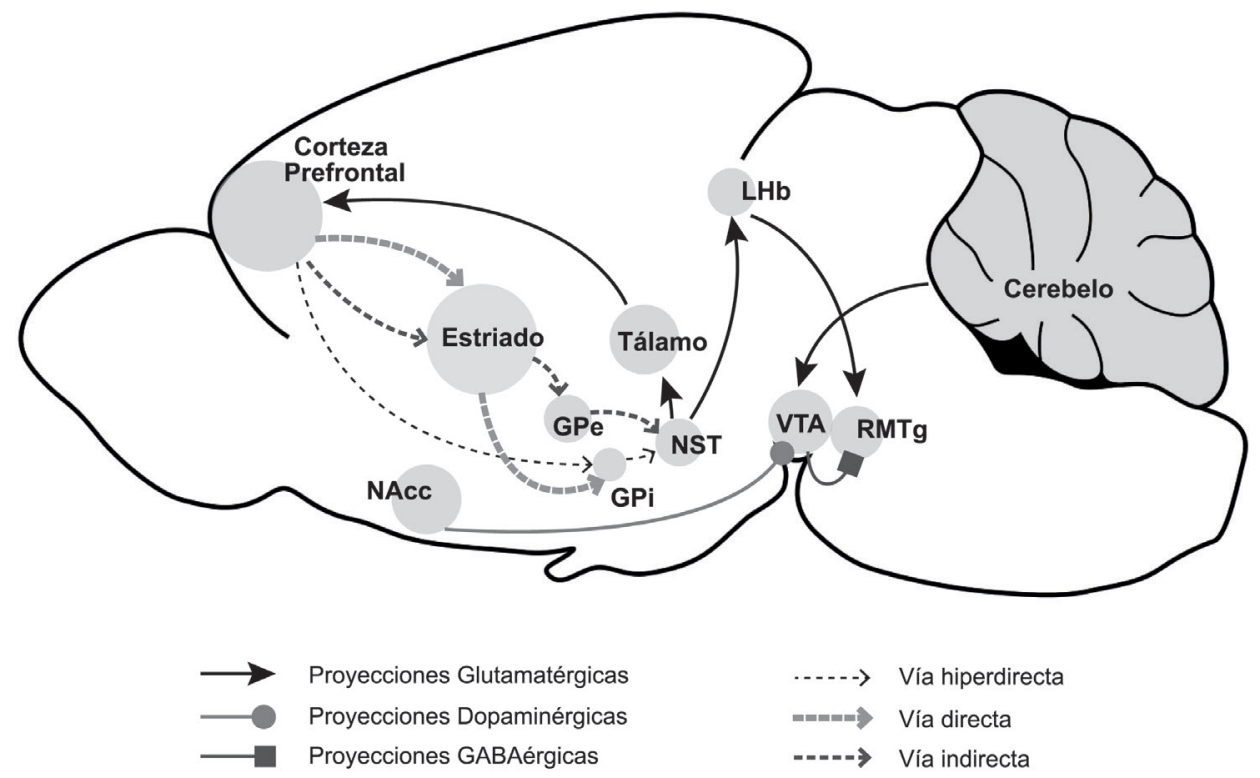

Nota: Corte sagital de cerebro de rata que muestra las vías que regulan el autocontrol, incluyendo la proyección del GPi (globo pálido interno) al HbL y del cerebelo al sistema de recompensa. Esta figura se ha construido a partir de la información de Carta et al., 2019; Koob \& Volkow, 2016; Lammel et al., 2012. Fue creada por el Diseñador Sergio Broseta por instrucciones de los autores y para los objetivos de esta publicación.

Recientemente se ha demostrado que el cerebelo $(\mathrm{Cb})$ proyecta directamente al ATV, promoviendo la liberación de DA, facilitando de esta manera la búsqueda del reforzador y la sensación subjetiva de placer (Carta et al., 2019) (Figura 2). Como observamos, el sistema del autocontrol comprende un cúmulo de estructuras que se comunican entre sí, conformando una red cortico-subcortical, que le asignan un valor reforzante al estímulo que puede ser positivo, y genera entonces un reforzamiento positivo o negativo, por lo que el estímulo puede funcionar como un reforzador negativo. La decisión de consumirlo o evitarlo depende de la experiencia; es decir, si el sujeto ha estado frente a este estímulo antes o no, pero también depende de la presión social y, en particular, de la presión de pares, que en el adolescente es crucial en la toma de decisiones.

La actividad de esta red cortico-subcortical del control inhibidor de la conducta ocurre principalmente por actividad GABAérgica y GLUérgica; sin embargo, estos sistemas están bajo la modulación ejercida directamente por el sECB, el cual tiene una amplia representación en el cerebro, como veremos a continuación.

Sistema de endocanabinoides (sECB). En las últimas cuatro decadas el sECB ha sido ampliamente estudiado. Se han descrito además del receptor a canabinoides 1 ( $\mathrm{CB} 1 \mathrm{R})$, el CB2R, sus agonistas endógenos, v. gr., anandamida (AEA), 2-araquidonilglicerol (2AG), oleamida (ODA), noladin éter, virodamina, n-araquidonil-dopamina y otros; además de las enzimas que participan en su síntesis y degradación, así como a los transportadores (Figura 3) (Méndez-Díaz et al., 2008). 
Figura 3. Sistema de Endocanabinoides
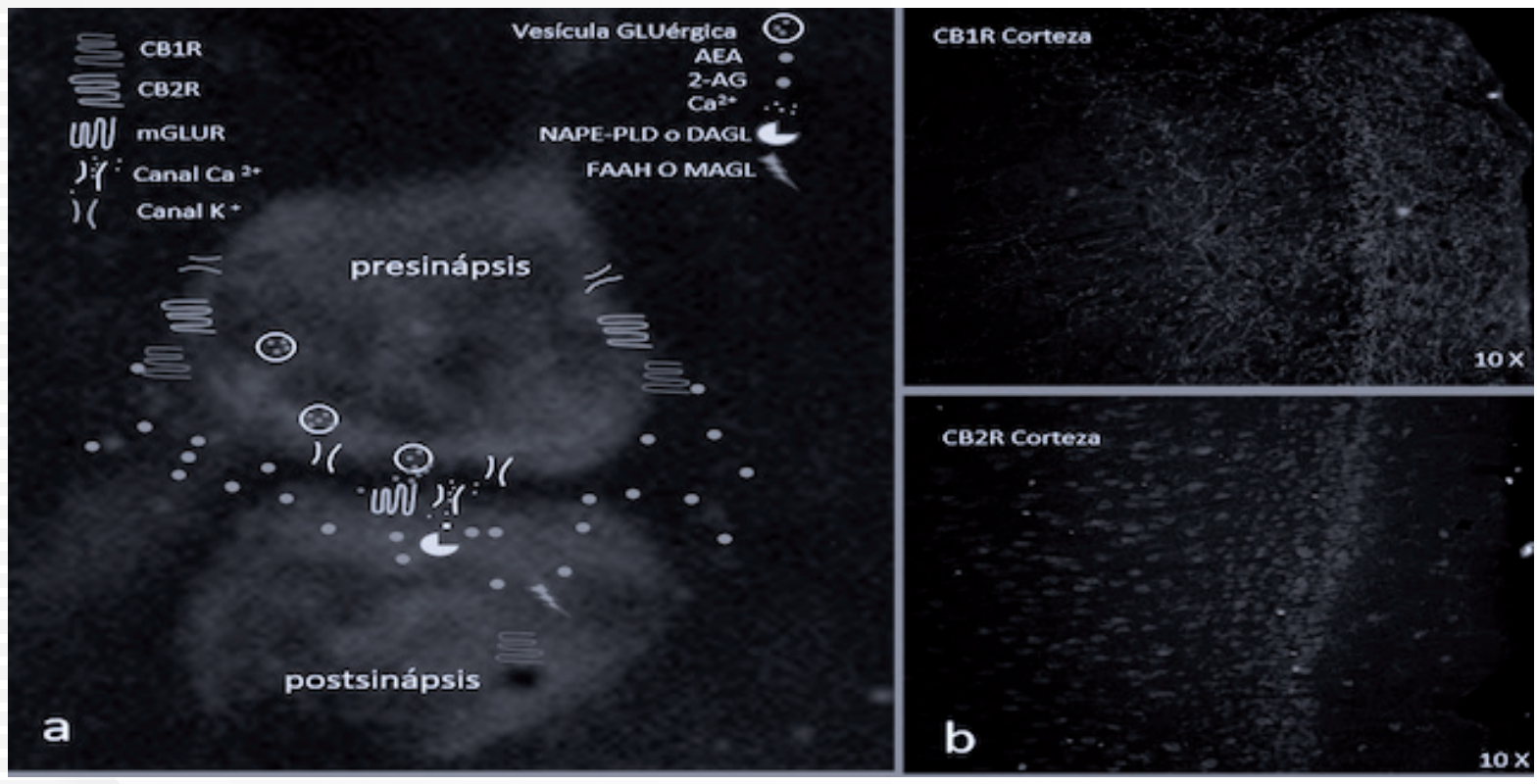

Nota: La imagen muestra una sinapsis: a) En la membrana de las neuronas postsinápticas se encuentran los precursores de endocanabinoides. Los eCB se sintetizan como consecuencia de un aumento de las concentraciones citosólicas de $\mathrm{Ca} 2+$, quien activa a las enzimas NAPE-PLD (sintetiza AEA) o DAG (sintetiza 2AG). Los eCB actúan como mensajeros retrógrados uniéndose a sus receptores presinápticos CB1R o $\mathrm{CB} 2 \mathrm{R}$, que inhiben a los canales de $\mathrm{Ca} 2+$ sensibles al voltaje y activan a los canales de $\mathrm{K}+$, generando una hiperpolarización de la membrana presináptica y disminuyendo la liberación del neurotransmisor. b) Inmunofluorescencia realizada en rebanadas de cerebro de rata para marcar la expresión de CB1R y CB2R en CPF. La inmunofluorescencia y fotografías mostradas fueron tomadas y procesadas por lo autores. La información de la figura a fue tomada de Méndez-Díaz et al., 2008 y Wȩdzony \& Chocyk, 2009.

El CB1R se expresa en neuronas y células gliales, de la corteza, neocorteza, hipocampo, putamen, globo pálido, NAc, AMI, ATV, locus coeruleus, área gris periacueductal (AGP), sustancia negra reticulada (SNr) y $\mathrm{Cb}$. En estas estructuras se encuentra principalmente expresado en neuronas GABAérgicas en contraste con las GLUérgicas (3:1 GABA/GLU). Por su parte, el CB2R se expresa en neuronas y células gliales de la corteza, hipocampo, cuerpo estriado, tálamo, HbL, AMI, SNr, AGP y $\mathrm{Cb}$. Ambos receptores están acoplados a una proteína $\mathrm{G}$

inhibidora (pG/i) (Méndez-Díaz et al., 2008; Wȩdzony \& Chocyk, 2009).

Se ha demostrado, en ratas, que la administración sistémica del antagonista a CB1, el SR141716A (Rimonabant), disminuye la búsqueda de cocaína (Filip et al., 2006) y de alcohol (Lallemand \& De Witte, 2006). Por lo que se ha propuesto al sECB como el sistema fundamentalmente afectado por la sustancias de abuso y que facilita la búsqueda y consumo de sustancias de abuso. De hecho, se ha observado que la administración de AEA en el EP de ratas disminuye la ingestión de alcohol, mientras que la administración del antagonista AM251 la incrementa (Méndez-Diaz et al., 2012). Adicionalmente, se ha encontrado, en ratas adolescentes, un comportamiento indiscriminado de búsqueda y alta motivación por alimento apetitoso, relacionado con una menor expresión de CB1R y CB2R en la CPF y el NAc. Estos rasgos están ausentes en las ratas adultas y viejas (Amancio-Belmont et al., 2017), confirmando la función del sECB de la CPF en el autocontrol. Con base en esta evidencia, sugerimos que el sECB es parte del sistema que regula el control del impulso y, por lo tanto, el consumo de sustancias.

Adicionalmente, el sECB ha sido involucrado en los mecanismos que subyacen algunas enfermedades mentales ademas del TCS. En 2005, Van Gaal et al. demostraron, en humanos, el efecto anorexigénico de Rimonabant. Sin embargo, este éxito se vio opacado por 
la inducción de síntomas psiquiátricos como depresión e ideación suicida. Actualmente la lista de trastornos psiquiátricos que involucran al sECB incluye además de depresión y ansiedad, psicosis, esquizofrenia, trastorno por estrés postraumático, trastorno por déficit de atención e hiperactividad, trastorno límite de personalidad y trastorno bipolar. A continuación, describiremos algunos trastornos psiquiátricos que se caracterizan por presentar impulsividad y TCS.

\section{Comorbilidad de Trastornos psiquiátricos con impulsividad y TCS}

Trastorno por déficit de atención e hiperactividad (TDAH). El TDAH es un trastorno que afecta al 5 y $12 \%$ de niños y adolescentes, respectivamente, y continúa en la edad adulta en el 60-70\% de los casos, lo que sugiere un 30-40 \% de remisión espontánea o con tratamiento (Ahmadalipour et al., 2019). Por sus caracteristicas clínicas puede ser con predomio de falta de atención, de hiperactividad e impulsividad o mixto. El TDAH puede presentar comorbilidad con otros trastornos psiquiátricos, como conducta disocial/antisocial, trastorno oposicionista desafiante y TCS. La comorbilidad de TCS y TDAH se estima que es del $45 \%$ (Wilens, 2004). Por otro lado, se ha sugerido que un polimorfismo de nucleótido único (snp) de la FAAH (2295633) (enzima que degrada a los ECB) podría ser un marcador de riesgo para el TDAH (Ahmadalipour et al., 2019). Aunque este es el primer estudio que muestra una relación la expresión de TDAH y el sECB, sugiere que es un marcador genético que vale la pena identificar para entender mejor el trastorno y esclarecer con fundamentos neuroquímicos la participación del sECB en TDAH y TUS.

Trastorno límite de la personalidad (TLP). Es un trastorno psiquiátrico que afecta al $2.7 \%$ de los adultos. Se caracteriza por estados de ánimo intensos y cambiantes, sentimiento de vacío, miedo al abandono, relaciones caóticas, estado de ánimo inestable y problemas de autoimagen e impulsividad (American Psychiatric Association, 2013). Alrededor del $78 \%$ de los adultos con TLP desarrollan TCS (Vest et al., 2018). Se ha demostrado que la concentración de AEA, detectada en el cabello de mujeres con diagnóstico de TLP, está disminuida en relación a mujeres sin TLP (Wingenfeld et al., 2018). Sin embargo, otros estudios reportan que la concentración en plasma se encuentra aumentada (Schaefer et al., 2014). Son ne- cesarios más estudios que determinen la participación del sECB en la fisiopatología del TLP y TCS.

Trastorno bipolar (TB). El TB se caracteriza por episodios inestables del estado de ánimo que oscilan entre la hipomanía/manía y depresión. Tiene una prevalencia estimada de $1.1 \%$ (Tipo I) y $1.6 \%$ (Tipo II) en la población general (Rote et al., 2018). La impulsividad está vinculada al curso de esta enfermedad. Se ha observado que los pacientes con TB Tipo I y comorbilidad de TCS muestran mayor impulsividad (Rote et al., 2018). En pacientes con TB Tipo I la prevalencia del consumo de sustancias de abuso es del 21-31\%, y existe una estrecha asociación entre un polimorfismo del gen que codifica para CB2R, el CNR2 524C>A y BD (Minocci et al., 2011). Demostrar que los pacientes con el polimorfismo CNR2 524C>A son más impulsivos, nos permite sugerir que el CB2R forma parte de los mecanismos del autocontrol en el TB.

Trastornos relacionados con ansiedad. Estos trastornos se caracterizan por presentar ansiedad y preocupación excesiva (anticipación aprensiva), que no corresponde con la situación que se vive, y que interfiere con la capacidad de funcionar normalmente. Los trastornos de ansiedad pueden contribuir a iniciar la dependencia de sustancias; los más asociados a TUS son principalmente el trastorno de pánico, síndrome de estrés postraumático, fobia social y agorafobia (Goodwin $\&$ Stein, 2013). En sujetos de 16 a 18 años de edad con algún trastorno de ansiedad, el $28.6 \%$ presentaron TCS, y de 18 a 21 años, el 40\% (Goodwin et al., 2004). Se ha observado que los sujetos que poseen el polimorfismo rs324420 de la FAAH, el cual se caracteriza por disminución de la actividad de la FAAH, manifiestan menos ansiedad (Lee et al., 2016). Esto sugiere que para mantener el control de la ansiedad es necesario un nivel de endocanabinoides que los sujetos ansiosos no alcanzan, posiblemente porque tienen una FAAH mas activa. Estos resultados han sido apoyados por evidencia farmacológica de que la autoadministración de canabis disminuye la ansiedad (Lintzeris et al., 2018).

Adicionalmente a estas enfermedades psiquiátricas, que se caracterizan por impulsividad, recientemente se ha descrito que el cerebelo $(\mathrm{Cb})$ esta involucrado en la expresión de la impulsividad. 
Disfunción del cerebelo. Se ha reportado que sujetos con TDAH, así como niños prematuros con sintomatología de impulsividad tienen un volumen cerebelar disminuido y una menor proporción de materia gris (GM) (Matthews et al., 2018). En pacientes alcohólicos en diferentes etapas de remisión, la disfunción frontocerebelosa parece ser un factor clave para predecir y explicar el déficit de autocontrol (Jung et al., 2014). Mediante estudios de neuroimagen, se ha demostrado sistemáticamente la activación cerebelosa cuando se presentan señales relacionadas con alguna droga (Jasinska et al., 2014). Cabe señalar que el $\mathrm{Cb}$ tiene una amplia expresión de ambos receptores para canabinoides, por lo que es posible que el sECB cerebelar este involucrado en la fisiología del control de los impulsos. Asimismo, los cambios que se han observado en la conectividad funcional en el cerebelo de los niños diagnosticados con TDAH pueden depender en parte del sECB (Jiang et al., 2019).

\section{Discusión}

A lo largo de la evolución, el ser humano adquirio el control de los impulsos como el elemento clave para la adaptación social y la sobrevivencia. Basta literatura señala que el fracaso social, académico, financiero y la expresión de diversos trastornos psiquiátricos, incluyendo los del consumo de sustancias (TCS), tienen como característica la impulsividad. El control de los impulsos sigue siendo el elemento clave del éxito en el sigo XXI.

Con base a sus carateristicas neuroanátomicas y conductuales, se ha descrito a los adolescentes como sujetos impulsivos y en consecuencia con vulnerabilidad a padecer algún TCS; sin embargo, en esta revisión se ha puesto en evidencia que ni el TCS ni la impulsividad son propias de los adolescentes. La proporcion de usuarios con TCS es incluso mayor en la población adulta (CONADIC, 2017), y vale la pena señalar que la impulsividad es el rasgo de la personalidad antisocial (propia del adulto) (Marzilli et al., 2021). Aunque podemos concluir que la impulsividad del adulto no es consecuencia del uso temprano de sustancias, la evidencia deja claro que la dupla impulsividad-uso de sustancias es exitosa para la expresión del TCS.

Por otro lado, se ha reponsabilizado a la CPF y las estructuras subcorticales del control del impulso como directoras del autocontrol; no obstante, aquí se ha propuesto agregar a este esquema a la $\mathrm{HbL}$ y el $\mathrm{Cb}$. Matthews et al. (2018) demuestran que la integridad del $\mathrm{Cb}$ es indispensable para la expresión de las conductas adaptativas, menor $\mathrm{Cb}$, mayor impulsividad. Ambas estructuras, regulan la actividad del ATV. Cuando el ATV activa al NAc motiva al individuo a buscar un reforzador y cuando el individuo consume, el reforzador incrementa aún más su actividad, generando la sensacion de placer (Kalivas \& Volkow, 2005). Es decir, ambos HbL y Cb están facilitando el comportamiento de búsqueda del reforzador, con base en su valor reforzante. En contraparte, ambos frenan la búsqueda del reforzador. De esta forma se insinúa la constitución de un sistema de motivaciónrecompensa-motor.

De los sistemas neuroquímicos que regulan este sistema de motivación-recompensa-motor, aquí se ha descrito a el sECB, quien aparece como un actor tanto del autocontrol como del TCS. Si bien la expresión de los receptores para canabinoides en estas estructuras es amplia (Méndez-Diaz et al., 2008) y en modelos animales se ha demostrado que su manipulación farmacologica modifica la expresión de las conductas impulsivas (Lallemand, \& De Witte, 2006; Méndez-Diaz et al., 2012; Amancio-Belmont et al., 2017), la muestra más evidente de su involucro la revelan los polimorfismos del sECB, presentes en las enfermedades psiquiátricas (TDAH, TLP, TB, ansiedad), por lo que deberían ser considerados como marcadores genéticos del TCS.

Pese a que es una desventaja nacer con alguno de estos trastornos psiquiatrícos, identificarlos y estudiarlos permitirá usarlos como una herramienta de diagnóstico y pronóstico para su prevención y tratamiento. Por ejemplo, las intervenciones cognitivo-conductuales para la prevención puede ayudar a los adolescentes a disminuir la impulsividad enseñandoles a identificar situaciones de riesgo (consumir una sustancia de abuso) y las señales interoceptivas que preceden a una acción impulsiva; la intervención cognitivo- conductual, para ayudar a los pacientes a manejar mejor los estados de ansia de consumo y prevenir recaídas; la atención plena o mindfulness, para reducir el ansia de consumo, al reducir el estrés, es decir, equilibrar la conectividad fronto-estriatal para el manejo de las emociones. 
En esta revisión se han descrito brevemente los mecanismos cerebrales involucrados en la vulnerabilidad a la adicción que incluyen la falta de integridad del sistema de control del impulso y la función del sECB. Se ha evidenciado la investigación en ciernes de la relación del sECB con enfermedades psiquiátricas que se caracterizan por ser una patología dual entre impulsividad y TCS. Sin embargo, resulta indispensable realizar una investigación básica que permita demostrar que la impulsividad y la busqueda de sustancias puede ser modulada farmacológicamente por canabinoides. En un futuro estos resultados podrían ser utilizados como estrategias farmacológicas para el control del TCS.

\section{Referencias}

Ahmadalipour, A., Mehdizadeh Fanid, L., Zeinalzadeh, N., Alizadeh, M., Vaezi, H., Hassanpour Aydinlou, Z., \& Noorazar, S. G. (2019). The first evidence of an association between a polymorphism in the endocannabinoid-degrading enzyme FAAH (FAAH rs2295633) with attention deficit hyperactivity disorder. Genomics, 112(2), 1330-1334. https:// doi.org/10.1016/j.ygeno.2019.07.024

Amancio-Belmont, O., Romano-López, A., Ruiz-Contreras, A. E., Méndez-Díaz, M., \& Prospéro-García, O. (2017). From adolescent to elder rats: Motivation for palatable food and cannabinoids receptors. Developmental Neurobiology, 77(8), 917-927. https://doi.org/10.1002/dneu.22472

American Psychiatric Association. (2013). Diagnostic and Statistical Manual of Mental Disorders (5th ed.). American Psychiatric Association. https://doi. org/10.1176/appi.books.9780890425596

Aron, A. R., \& Poldrack, R. A. (2006). Cortical and subcortical contributions to stop signal response inhibition: Role of the subthalamic nucleus. Journal of Neuroscience, 26(9), 2424-2433. https://doi. org/10.1523/JNEUROSCI.4682-05.2006

Baik, J. H. (2013). Dopamine signaling in reward-related behaviors. In Frontiers in Neural Circuits (Vol. 7, Issue OCT). Front Neural Circuits. https://doi. org/10.3389/fncir.2013.00152

Barrot, M., Sesack, S. R., Georges, F., Pistis, M., Hong, S., \& Jhou, T. C. (2012). Braking dopamine systems:
A new GABA master structure for mesolimbic and nigrostriatal functions. Journal of Neuroscience, 32(41), 14094-14101. https://doi.org/10.1523/ JNEUROSCI.3370-12.2012

Carta, I., Chen, C. H., Schott, A. L., Dorizan, S., \& Khodakhah, K. (2019). Cerebellar modulation of the reward circuitry and social behavior. Science, 363(6424). https://doi.org/10.1126/science. aav0581

CONADIC. (2017). Encuesta Nacional de Consumo de Drogas, Alcohol y Tabaco, ENCODAT 2016-2017 | Comisión Nacional contra las Adicciones | Gobierno | gob.mx. https://www.gob.mx/salud\%7Cconadic/ acciones-y-programas/encuesta-nacional-deconsumo-de-drogas-alcohol-y-tabaco-encodat-2016-2017-136758

Dumontheil, I., Burgess, P. W., \& Blakemore, S. J. (2008). Development of rostral prefrontal cortex and cognitive and behavioural disorders. In Developmental Medicine and Child Neurology (Vol. 50, Issue 3, pp. 168-181). Dev Med Child Neurol. https:// doi.org/10.1111/j.1469-8749.2008.02026.x

ENSANUT. (2018). Encuesta Nacional de Salud y Nutrición 2018 Presentación de resultados. 4ta Edición, 1-42. https://www.inegi.org.mx/contenidos/programas/ensanut/2018/doc/ensanut_2018_presentacion_resultados.pdf

Filip, M., Gołda, A., Zaniewska, M., McCreary, A. C., Nowak, E., Kolasiewicz, W., \& Przegaliński, E. (2006). Involvement of cannabinoid CB1 receptors in drug addiction: Effects of rimonabant on behavioral responses induced by cocaine. Pharmacological Reports, 58(6), 806-819.

García-Molina, A., Enseñat-Cantallops, A., Tirapu-Ustárroz, J., \& Roig-Rovira, T. (2009). Maduración de la corteza prefrontal y desarrollo de las funciones ejecutivas durante los primeros cinco años de vida. In Revista de Neurologia (Vol. 48, Issue 8, pp. 435-440). Revista de Neurologia. https://doi. org/10.33588/rn.4808.2008265

Goodwin, R. D., Fergusson, D. M., \& Horwood, L. J. (2004). Association between anxiety disorders and substance use disorders among young persons: Results of a 21-year longitudinal study. Jour- 
nal of Psychiatric Research, 38(3), 295-304. https:// doi.org/10.1016/j.jpsychires.2003.09.002

Goodwin, R. D., \& Stein, D. J. (2013). Anxiety disorders and drug dependence: Evidence on sequence and specificity among adults. Psychiatry and Clinical Neurosciences, 67(3), 167-173. https://doi. org/10.1111/pcn.12030

Institute for Health Metrics and Evaluation. (2018). Findings from the Global Burden of Disease Study 2017. The Lancet, 27. http:/www.healthdata.org/ policy-report/findings-global-burden-diseasestudy-2017

Jasinska, A. J., Stein, E. A., Kaiser, J., Naumer, M. J., \& Yalachkov, Y. (2014). Factors modulating neural reactivity to drug cues in addiction: A survey of human neuroimaging studies. In Neuroscience and Biobehavioral Reviews (Vol. 38, Issue 1, pp. 1-16). Elsevier Ltd. https://doi.org/10.1016/j.neubiorev.2013.10.013

Jiang, K., Yi, Y., Li, L., Li, H., Shen, H., Zhao, F., Xu, Y., \& Zheng, A. (2019). Functional network connectivity changes in children with attentiondeficit hyperactivity disorder: A resting-state fMRI study. International Journal of Developmental Neuroscience, 78, 1-6. https://doi.org/10.1016/j. ijdevneu.2019.07.003

Jung, Y. C., Schulte, T., Müller-Oehring, E. M., Namkoong, K., Pfefferbaum, A., \& Sullivan, E. V. (2014). Compromised frontocerebellar circuitry contributes to nonplanning impulsivity in recovering alcoholics. Psychopharmacology, 231 (23), 4443-4453. https://doi.org/10.1007/s00213014-3594-2

Kalivas, P. W., \& Volkow, N. D. (2005). The neural basis of addiction: A pathology of motivation and choice. In American Journal of Psychiatry (Vol. 162, Issue 8, pp. 1403-1413). Am J Psychiatry. https:// doi.org/10.1176/appi.ajp.162.8.1403

Koob, G. F., \& Volkow, N. D. (2010). Neurocircuitry of addiction. In Neuropsychopharmacology (Vol. 35, Issue 1, pp. 217-238). Nature Publishing Group. https://doi.org/10.1038/npp.2009.110

Koob, G. F., \& Volkow, N. D. (2016). Neurobiology of addiction: a neurocircuitry analysis. In The Lancet
Psychiatry (Vol. 3, Issue 8, pp. 760-773). https:// doi.org/10.1016/S2215-0366(16)00104-8

Lallemand, F., \& De Witte, P. (2006). SR147778, a CB1 cannabinoid receptor antagonist, suppresses ethanol preference in chronically alcoholized Wistar rats. Alcohol, 39(3), 125-134. https://doi. org/10.1016/j.alcohol.2006.08.001

Lammel, S., Lim, B. K., Ran, C., Huang, K. W., Betley, M. J., Tye, K. M., Deisseroth, K., \& Malenka, R. C. (2012). Input-specific control of reward and aversion in the ventral tegmental area. Nature, 491(7423), 212-217. https://doi.org/10.1038/ nature 11527

Lee, T. T. Y., Hill, M. N., \& Lee, F. S. (2016). Developmental regulation of fear learning and anxiety behavior by endocannabinoids. In Genes, Brain and Behavior (Vol. 15, Issue 1, pp. 108-124). Blackwell Publishing Ltd. https://doi.org/10.1111/ gbb. 12253

Lintzeris, N., Driels, J., Elias, N., Arnold, J. C., McGregor, I. S., \& Allsop, D. J. (2018). Medicinal cannabis in Australia, 2016: The cannabis as medicine survey (CAMS-16). Medical Journal of Australia, 209(5), 211-216. https://doi.org/10.5694/ mja17.01247

Marzilli, E., Cerniglia, L., \& Cimino, S. (2021). Antisocial Personality Problems in Emerging Adulthood: The Role of Family Functioning, Impulsivity, and Empathy. Brain Sciences, 11 (6), 687. doi: 10.3390/ brainsci11060687.

Matthews, L. G., Inder, T. E., Pascoe, L., Kapur, K., Lee, K. J., Monson, B. B., Doyle, L. W., Thompson, D. K., \& Anderson, P. J. (2018). Longitudinal Preterm Cerebellar Volume: Perinatal and Neurodevelopmental Outcome Associations. Cerebellum, 17(5), 610-627. https://doi.org/10.1007/ s12311-018-0946-1

Méndez-Díaz, M., Herrera-Solís, A., Soria-Gómez, E., Rueda-Orozco, P., \& Prospéro-García, O. (2008). Mighty cannabinoids: A potential pharmacological tool in medicine. Neural Mechanisms of Action of Drugs of Abuse and Natural Reinforcers., 661(2), 137-157. https://www.researchgate.net/publication/259739126 
Méndez-Díaz, M., Romero Torres, B., Cortés Morelos, J., Ruíz-Contreras, A., \& Prospéro García, O. (2017). Neurobiología de las adicciones. Revista de La Facultad de Medicina (México), 60(1), 6-16. https:/www.medigraphic.com/cgi-bin/new/resumen.cgi? IDARTICULO $=70500$

Méndez-Díaz, M., Rueda-Orozco, P. E., Ruiz-Contreras, A. E., \& Prospéro-García, O. (2012). The endocannabinoid system modulates the valence of the emotion associated to food ingestion. Addiction Biology, 17(4), 725-735. https://doi.org/10.1111/ j.1369-1600.2010.00271.x

Minocci, D., Massei, J., Martino, A., Milianti, M., Piz, L., Di Bello, D., Sbrana, A., Martinotti, E., Rossi, A. M., \& Nieri, P. (2011). Genetic association between bipolar disorder and 524A $>C$ (Leu133Ile) polymorphism of CNR2 gene, encoding for CB2 cannabinoid receptor. Journal of Affective Disorders, 134(1-3), 427-430. https://doi.org/10.1016/j. jad.2011.05.023

Rote, J., Dingelstadt, A. M. L., Aigner, A., Bauer, M., Fiebig, J., König, B., Kunze, J., Pfeiffer, S., Pfennig, A., Quinlivan, E., Simhandl, C., \& Stamm, T. J. (2018). Impulsivity predicts illness severity in long-term course of bipolar disorder: A prospective approach. Australian and $\mathrm{New}$ Zealand Journal of Psychiatry, 52(9), 876-886. https://doi. org/10.1177/0004867418783062

Schaefer, C., Enning, F., Mueller, J. K., Bumb, J. M., Rohleder, C., Odorfer, T. M., Klosterkötter, J., Hellmich, M., Koethe, D., Schmahl, C., Bohus, M., \& Leweke, F. M. (2014). Fatty acid ethanolamide levels are altered in borderline personality and complex posttraumatic stress disorders. European Archives of Psychiatry and Clinical Neuroscience, 264(5), 459-463. https://doi.org/10.1007/ s00406-013-0470-8

Tirapu-Ustárroz, J., García-Molina, A., Luna-Lario, P., Roig-Rovira, T., \& Pelegrín-Valero, C. (2008). Modelos de funciones y control ejecutivo (II). In Revista de Neurologia (Vol. 46, Issue 12, pp. 742-750). https://doi.org/10.33588/rn.4612.2008252

Van Gaal, L. F., Rissanen, A. M., Scheen, A. J., Ziegler, O., \& Rössner, S. (2005). Effects of the cannabinoid-1 receptor blocker rimonabant on weight reduction and cardiovascular risk factors in overweight patients: 1-Year experience from the RIO-Europe study. Lancet, 365(9468), 1389-1397. https://doi. org/10.1016/S0140-6736(05)66374-X

Velázquez, J. A. V., Icaza, M. E. M. M., del Campo Sánchez, R. M., Ito, D. A. F., Gamiño, M. N. B., Escobar, E. R., Salazar, R. M., Cirett, M. B., Hernández, I. S. S., \& Martínez, V. C. (2016). Drug use among students in Mexico: Trends and magnitude of the problem. Salud Mental, 39(4), 193-203. https:// doi.org/10.17711/SM.0185-3325.2016.023

Vest, N. A., Murphy, K. T., \& Tragesser, S. L. (2018). Borderline personality disorder features and drinking, cannabis, and prescription opioid motives: Differential associations across substance and sex. Addictive Behaviors, 87, 46-54. https://doi.org/10.1016/j. addbeh.2018.06.015

Wędzony, K., \& Chocyk, A. (2009). Cannabinoid CB1 receptors in rat medial prefrontal cortex are colocalized with calbindin- but not parvalbumin- and calretinin-positive GABA-ergic neurons. Pharmacological Reports, 61(6), 1000-1007. https://doi. org/10.1016/S1734-1140(09)70161-6

Wilens, T. E. (2004). Attention-deficit/hyperactivity disorder and the substance use disorders: The nature of the relationship, subtypes at risk, and treatment issues. In Psychiatric Clinics of North America (Vol. 27, Issue 2, pp. 283-301). Psychiatr Clin North Am. https://doi.org/10.1016/S0193953X(03)00113-8

Wingenfeld, K., Duesenberg, M., Fleischer, J., Roepke, S., Dziobek, I., Otte, C., \& Wolf, O. T. (2018). Psychosocial stress differentially affects emotional empathy in women with borderline personality disorder and healthy controls. Acta Psychiatrica Scandinavica, 137(3), 206-215. https://doi. org/10.1111/acps.12856 